Canadian

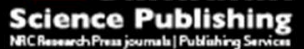

Canadian Journal of Civil Engineering Revue canadienne de génie civil

\title{
Fracture Characteristics of Laboratory-Tested Soda Lime Glass Specimens
}

\begin{tabular}{|r|l|}
\hline Journal: & Canadian Journal of Civil Engineering \\
\hline Manuscript ID & cjce-2016-0374.R1 \\
\hline Manuscript Type: & Article \\
\hline Date Submitted by the Author: & $14-$-Nov-2016 \\
\hline Complete List of Authors: & $\begin{array}{l}\text { Yankelevsky, David; Technion Israel Institute of Technology Faculty of Civil } \\
\text { and Environmental Engineering } \\
\text { Spiller, Kevin; University of Toronto } \\
\text { Packer, Jeffrey; University of Toronto, Civil Engineering } \\
\text { Seica, Michael; University of Toronto }\end{array}$ \\
\hline Keyword: & $\begin{array}{l}\text { material properties (MP) < Engineering Materials, fracture; fatigue and } \\
\text { dam < Engineering Mechanics, experimental research < type of paper to } \\
\text { review }\end{array}$ \\
\hline & \multicolumn{2}{|c}{} \\
\hline
\end{tabular}




\section{Fracture Characteristics of Laboratory-Tested Soda Lime Glass Specimens}

\section{David Z. Yankelevsky}

Faculty of Civil \& Environmental Engineering, Technion-Israel Institute of Technology, Haifa 32000, Israel;

On Sabbatical Leave, Department of Civil Engineering, University of Toronto, Toronto, Ontario M5S 1A4, Canada

\section{Kevin Spiller, Jeffrey A. Packer ${ }^{+}$}

Department of Civil Engineering, University of Toronto, Toronto, Ontario M5S 1A4, Canada

\section{Michael V. Seica}

Explora Security Ltd., London SW6 2BW, U.K.;

Department of Civil Engineering, University of Toronto, Toronto, Ontario M5S 1A4, Canada

${ }^{+}$Corresponding author: Tel: (416) 978 4776; E-mail: jeffrey.packer@utoronto.ca 


\begin{abstract}
Static testing of glass plates has shown a pronounced distribution in the plate strength. The origin of fracture location along the plate span, the corresponding critical tensile stress and the size of the critical flaw at the origin of fracture are failure characteristics of major importance. This paper provides data and insight into parameters influencing these failure characteristics through laboratory testing and analysis of soda lime glass, to find a rationale behind the observed disorder of their magnitudes and distributions.

A recent stochastic model for glass plate analysis is implemented to predict the magnitudes and distributions of the failure characteristics, and to compare these with laboratory tests. The model provides good predictions of the distributions and magnitudes of the failure characteristics and sheds light on glass plate behaviour. It demonstrates that there are mechanics-based explanations to the wide scatter of experimental results and to the apparent disorder in glass behaviour.
\end{abstract}

Keywords: glass, four-point bending, standards, modeling, tensile strength, origin of fracture, laboratory testing, probabilistic distribution, brittle failure, critical flaw 


\section{Introduction}

Glass is an important material that is used widely in the construction sector. It is used in window panes, curtain walls, vitrines, and even in special structures like bridges, inspection decks, staircases, etc. (Ledbetter et al. 2006). This study is focused on soda lime annealed glass that is a widely used common product for window glazing. It is made of well-defined materials (EN-572-1 2004), and some mechanical properties like the Young's modulus and Poisson's ratio are quite well known. As maintenance and weathering affect the glass properties, as-received glass (henceforth denoted as new glass) is considered in this study to account for the natural properties only and exclude any other effect on its behaviour.

When two similar glass plates are tested under similar support conditions, and they are subjected to similar static loading, their failure characteristics are often very different (Dalgliesh et al. 1990; Norville et al. 1993). The system of cracks may look entirely different, the origin of fracture (OF) is likely to be located at different coordinates and the magnitude of the critical loading that causes fracture is also different. The tensile strength in bending depends on the plate geometry, on the support conditions and on the loading setup. The magnitude of the ultimate loading and the location of the OF are key parameters for determination of the critical tensile stress at which fracture occurs. The magnitude of the critical stress at failure is different in every tested plate as a result of the above.

The strength determination of glass is performed according to standard tests in which a rather small number of some 30 specimens, of a standard size, are subjected to a standard loading setup. Loading of these one-way plates is performed under standard specified controlled conditions and the fracture load is determined. From these data it is expected to determine the experimental glass strength. Data compilation of all standard test series shows 
a pronounced distribution of the plate strengths, with a spread of even $30-50 \%$ of the mean strength (Veer et al. 2001). Commonly, it is the critical tensile strength at failure which is reported, and attempts have been made to model its distribution by existing well-known functions like the Weibull distribution (Beason 1980; Beason et al. 1984). For different test series, different parameters of the Weibull function yielded the best fit (Beason et al. 1984; Brown 1972; Sedlacek et al. 1995); this indicates that there are no universal Weibull constants to fit the different glass test data. Moreover, recent studies indicated that other common statistical functions like the Normal or the Log-normal distribution functions show an even better fit to some experimental data (Norhuda et al. 2010; Leon 2001). This leads to a need to examine the distribution characteristics without assigning any predefined statistical function (Yankelevsky 2014).

Plate fracture is initiated at the OF, the location of which may be rather distant from the cross section at which a maximum bending moment is developed and in one-way plates it may be located at different points across the plate width. It seems that the OF may be located almost at any point on the plate surface, depending on the applied loading system, and it cannot be a priori predicted. Although standards focus on the strength and do not refer to the location of the OF, the latter is one of the plate's failure characteristics that is as important, because its location is related to the magnitude of the applied stresses at failure. Therefore, it is interesting and important to gain insight into this aspect as well as into the corresponding critical tensile strength at failure and the other related failure characteristics, and to examine the apparent disorder of the OF location and the magnitudes of other parameters.

Recently, a new stochastic model for glass plate analysis has been developed (Yankelevsky 2014) and it is capable of predicting the strength distribution of a sample of similarly loaded 
glass plates and the locations of their OF, based on an assumed distribution of flaws and without pre-assigning a common statistical distribution function.

The model is based on the assumption of numerous randomly distributed flaws on the glass plate surface and underneath the surface. In general, these flaws may originate from production and maintenance procedures, however in the case of new glass that is investigated here, only production flaws are considered, and all other possible sources of defects and flaws due to maintenance, handling, weathering, etc., are excluded. It is assumed that each flaw behaves like a small embedded crack that expands abruptly upon loading at its critical stress. The latter depends on the flaw characteristics. This new model (Yankelevsky 2014) refers to the flaw distribution and evaluates the corresponding critical stresses and the OF locations. The critical stresses at all flaws are compared with the applied stresses that result from the applied loading system, for a given plate geometry and boundary conditions. This enables the calculation of the coordinates of the OF and the critical stress that causes that fracture in each analyzed plate and a comparison of the probabilistic distribution of a sample of specimens with the test data.

This model is implemented in the present study to examine the test results of a sample of new soda lime glass standard-size specimens that were subjected to a standard four-point bending loading system. The comparison of the model predictions with the test results, and further investigation with the model, is most helpful to examine the model reliability, on the one hand, and to gain insight into glass plate behaviour and its major characteristics on the other hand. 


\section{Characterization of the critical flaw parameters}

Consider a typical standard bending test, as mentioned above, that is conducted under standard specified conditions. Loading is monotonically increased at a constant loading rate until a sudden failure occurs. Several parameters pertaining to the failure may be recorded and analyzed:

a. The Location of the Critical Flaw - At the end of a typical test, the fragments of the fractured specimen are collected and assembled to restore the original specimen geometry, and the width and length coordinates of the fracture origin, where the critical flaw is activated, are measured. Two major parameters are obtained: the width coordinate that is measured in the specimen cross section containing the OF, indicates also whether the failure occurred on the specimen's face or along its edge. In the latter case, it may be argued that an edge failure may have occurred due to a flaw produced or affected by the specimen's cutting process; as opposed to a face flaw failure which is not attributable to cutting and processing. To avoid the possible cutting effect on the results, the edge failure results can be excluded from the other results and examined separately to enable strength analysis of the face failure cases only. The length coordinate of the OF location determines the location of the critical section along the specimen axis at which failure occurs. This coordinate is necessary to determine the critical stress, at the OF location, as detailed in the following.

b. The Critical Stress - With the known length coordinate of the OF location, as well as the data regarding the loading setup and magnitude of the critical load, the bending moment at the critical cross section perpendicular to the specimen axis may be determined. Considering one-way bending of the specimen under a four-point bending loading, a constant stress distribution across the specimen's width may be assumed and knowing the cross- 
section dimensions of the critical cross section yields the critical tensile stress at fracture of the tested specimen.

c. The Size of the Critical Flaw - A direct approach to determine the size of the critical flaw may be suggested through mapping of the flaws across the entire specimen area prior to performing the test and identifying the specific critical flaw at which failure was initiated by using the recorded coordinates of the OF location. This is an exhaustive procedure that needs to be repeated for each tested specimen, and moreover current technology does not offer a satisfying solution for its implementation. Alternatively, an indirect technique may be used to assess the size of the critical flaw. This is based on a post-test fractographic investigation of the fractured surface as described in the following. Assessing the size of the critical flaw is an important part of the overall clarification of the entire glass plate behaviour.

Earlier fractographic studies on glass show that the fracture-initiating flaw is surrounded by a smooth mirror zone that is defined by the mirror radius, $r_{M}$. A mist zone is located between the hackle (defined by the hackle radius $r_{H}$ ) and the mirror zones (Figure 1) (Mechnolsky 1974).

It has been shown that the there exists a constant ratio between the mirror radius $r_{M}$ and the critical flaw radius $\mathbf{r}_{\mathbf{F}}$ (or crack half length, b)( Mechnolsky 1974). For soda lime glass it is:

$$
r_{M} \approx 10 r_{F}
$$

Also, it has been extensively demonstrated that the product of strength and the square root of the mirror radius $r_{M}$ is constant (Mechnolsky 1974). Thus, from measurement of the mirror radius, the radius of the critical flaw may be assessed and the critical stress may be determined. 
In the following section, an extensive test program is described in which results of the above-discussed major parameters are described.

\section{Laboratory Tests on standard specimens}

\subsection{General}

In order to study aspects of failure initiation in glass specimens, and especially focus on the major failure characteristics of the OF, the critical stress and the critical flaw size, a series of tests should be conducted. It is advantageous to follow a certain standard that is used to determine the strength of glass and to perform additional measurements of the OF coordinates and the radius of the critical flaw size, that are not required by the standard. The most common and simple standard tests for glass plate strength determination are four-point bending tests, that apply one-way bending to glass specimens. It is a simple test that is easy to implement in the laboratory. There exist two major standards for four-point bending: The European Standard EN 1288-3 (2000) that refers to relatively large span (1000 mm) specimens with width of $360 \mathrm{~mm}$, and the ASTM C158-02 (2012) that specifies smaller specimens with a span of $200 \mathrm{~mm}$ and a nominal width of $38.1 \mathrm{~mm}$. The latter standard seems to be more practical as it can be conducted under modest laboratory conditions and consumes smaller amounts of material, thus allowing tests on more specimens than the minimum required (30 specimens) for a reasonable effort and cost.

Tests were therefore carried out on $250 \times 38 \mathrm{~mm}$ plates with a span of $200 \mathrm{~mm}$, following all the standard requirements as specified in ASTM C158-02 (2012). All specimens were cut from larger, approximately $12 \mathrm{~mm}$ thick, new glass panes using the score-and-break technique. Each specimen was then wrapped and sealed to protect it from any damaging effect and to 
isolate it from temperature and humidity influences. It was unwrapped before its placement on the test setup supports. The test setup is shown in Figure 2. All tests were performed using the same load frame and machined steel supports. Supports were free to rotate about two axes to eliminate the potential for uneven loading.

Test specimens were placed symmetrically across the supports where the specimen extended $25 \mathrm{~mm}$ beyond the support on each side of the specimen. The specimen orientation was such that the surface, which was scored during the cutting process, was at the top, and upon bending it was thus in compression. This reduces a possible effect of the cutting process and fabrication-induced cracks, on the test results.

A safety precaution against glass fragments that may be released during fracture was devised. A metal box was constructed (Figure 3) to fit on top of the bottom supports and enclose the specimen during testing. The closed volume compartment also maintained constant climatic conditions. The tests were conducted under controlled climatic conditions: temperature $\left(24^{\circ} \mathrm{C}\right)$ and a relatively low humidity $(32.4 \%)$. These conditions are in agreement with the ASTM C518-02(2012) standard requirements (temperature $23 \pm 2^{\circ}$ and humidity $40 \pm 10 \%)$. The EN $1288-03$ standard allows a wider range of temperature and humidity $\left(23 \pm 5^{\circ}\right.$ and $40 \%-70 \%)$. A Plexiglas window in front of the box enabled side-on inspection of the test in progress.

Loading was applied at a uniform rate and was displacement-controlled. The displacement rate was about $0.012 \mathrm{~mm} / \mathrm{s}$ to achieve the requisite stress rate of approximately $1.1 \mathrm{MPa} / \mathrm{s}$ as required by ASTM C158-02 (1.1 $\pm 0.2 \mathrm{MPa} / \mathrm{sec})$. The test instrumentation included an LVDT that was mounted underneath the test specimens, which was located at mid-span, and a load 
cell for force measurements, the readings of which were compared with the readings of the test frame load cell for accuracy.

Upon loading the glass plate a linear force-displacement curve was observed on the LVDT reading until fracture, indicating the linear elastic brittle behaviour of the tested plates. At a certain stage of loading the plate reached its ultimate strength unexpectedly, and immediately broke into several large shards and more small pieces around the location of the OF. At the end of each test, the pieces were collected and assembled to restore the original shape of the specimen and its crack pattern just prior to failure.

The OF was identified in each specimen. One can classify the fracture origins as either an edge failure, where the OF lies along the plate's longitudinal edge, or a face failure, where the OF is located at the bottom surface of the plate, at a distance from the edge. The two different types of edge and face failure are shown in Figure 4.

As was mentioned above, face failure cases are examined separately to avoid the possible cutting effect on causing edge failure and affecting the results.

\subsection{Four-Point Bending Test Results}

A total of 83 specimens were tested in four-point bending in accordance with ASTM C15802 (2012); 56 of them underwent face failure. This sample size is considerably larger than the minimum number of 30 specimens that is required by the standard ASTM C158-02 (2012).

The major experimental failure characteristics of the face failure specimens are detailed in the following:

a. The Location of the Origin of Fracture - The measured longitudinal coordinate from the specimen end is given in Table 1 (column 3 ) and its distribution is shown in Figure 5. 
The OF locations are widely spread along the plate and across its width. They ranged within and beyond the load span of $100 \mathrm{~mm}$, from a short distance somewhat to the left of the lefthand-side load and to the right of the right-hand-side load, as can be seen in the Figure 5 . The density varies along the load span; this is a typical variation in relatively small sample sizes, whereas it is expected that in very large sample sizes an almost constant density will appear along the load span. The observed density variation in these test series looks arbitrary and shows no special significant trend. Different repeated tests of other samples are likely to show different distributions.

b. The Critical Tensile Bending Stress at Fracture - The magnitude of the tensile bending stress at failure for each specimen was calculated with the specific data of the critical load at failure, the location of the OF and the critical cross-section geometry, for which the width and height of the critical cross section were measured. The calculated stresses are given in Table 1 (column 2) and the distribution of their magnitude is shown in Figure 6.

The tensile stress at failure varies widely between 53MPa and $129 \mathrm{MPa}$ with a mean stress of 79.63MPa. This is a significant range between the smallest and largest stress magnitudes, with a variation of $\sim \pm 48 \%$ from the mean stress. Although the distribution indicates a higher density around $80 \mathrm{MPa}$ and lower densities at the lower and higher stress range ends, the distribution shape is far from having a smooth bell-shaped distribution that would be expected for a considerably larger sample of specimens and its variation looks unpredictable.

c. Crack Pattern - Figure 7 shows bottom views of several typical crack patterns of restored specimens after failure, with different locations of the fracture origin. The crack patterns are entirely different in every specimen, in their configuration and OF location. 
In this loading case it is also noticed that the fracture origin may be located at almost any point along the plate axis and across its width, except for the vicinity of the supports. The crack patterns and the shapes of fractured plates are very different from each other.

d. Size of Critical Flaw - Fractograpic microscopic measurements of the fractured specimens were carried out and typical pictures of the fracture origin were obtained (Figure 8). Measurements of the mirror radius were taken for all tested specimens (Table 1, column 4). Following the procedure described in section 2-c above, the approximate radius of the critical flaw was calculated (Table 1, column 5). A very wide range of critical flaw sizes was measured with critical flaw radii varying between 23-178 $\mu \mathrm{m}$.

e. Relationship between the Critical Values of the Tensile Stress and the Flaw Radius -

The critical stress and the critical flaw size have been determined independently (sections 3.2b and 3.2-d above). In this section their inter-relationship will be investigated.

It has been found (Mechnolsky et al. 1974) that the product of glass strength and the square root of the mirror radius yields a constant value $A$ :

$$
\sigma \cdot \sqrt{r_{M}}=A
$$

For soda lime glass the value of A is about 2 (Mechnolsky et al. 1974; Marshall et al. 1980).

Table 1 (column 6 ) shows the calculated value of the constant $A$ for this series of tests. An average of 2.05 is obtained with a slight variation around the average; the calculated standard deviation is $0.1 \mathrm{MPa}-\mathrm{m}^{1 / 2}$. The distribution of the critical flaw radii, as derived from the test series, is shown in Figure 9. 


\section{The Model}

\subsection{General}

A new stochastic model has been recently developed (Yankelevsky 2014). With this model numerical simulation of a sample of glass plates may be conducted and the statistical strength of each specimen as well as the strength distribution of the sample may be predicted. The model also calculates other characteristics of the failure. Thus, it enables examination of the tested specimens through numerical simulation in an attempt to predict the test data and gain insight into the behaviour of these glass plates. Its comparisons with different test data has shown very good agreement and shed light on different characteristics of the behaviour of glass plates (Yankelevsky 2014). Such a model is needed to examine whether there are clear trends of behaviour behind the observed test results that are reported above. This examination is carried out in section 5.

For the sake of completeness, several key features of the model are presented in the following sections. In general, the model assumes that glass behaves as a linear elastic brittle material that fractures spontaneously at a load or stress level that cannot be a priori determined. It assumes that the micro structure of a glass plate contains numerous flaws of different sizes, both surface and in-depth flaws. Fracture occurs when a critical flaw is subjected to its corresponding critical stress and it is likely that it is a surface or near-surface flaw located at the tensile-stressed side of the plate. The model is composed of three major parts: the properties of a single flaw, the population of flaws in a specimen and the algorithms to analyze the location of the critical flaw and at what loading level failure occurs. These parts are briefly described in the following.

\subsection{The critical flaw}


Numerous tests including the above-described series of tests demonstrate that glass is a brittle material that behaves as an elastic solid at fracture. It is assumed that fracture of glass initiates at the location of a crack that first opens up due to the stresses acting on it. This is the critical crack.

Due to the distribution of flaws the critical crack is not necessarily located at the point of maximum tensile stress. Based on earlier works (Griffith 1920; Irwin 1962), a well-known expression correlating the failure stress $\sigma$ and half length of the critical crack "b" is given by:

$K_{I}=Y \sigma \sqrt{\pi b}$

Where $\mathrm{Y}$ is a geometrical factor, depending on the flaw shape; for example: 1.12 for a general surface crack (Irwin 1962), 0.713 for half-penny shaped crack on a flexural element (Overend and Zammit 2012) and 0.637 for an elliptical crack (Lawn 1993). Failure occurs when the stress intensity factor reaches its critical value $K_{l c}$ (fracture toughness), the value of which varies within the range of $(0.72-0.82) \mathrm{MPa}-\mathrm{m}^{1 / 2}$ for soda lime glass. Commonly a value of 0.75 MPa- $\mathrm{m}^{1 / 2}$ is used (Overend and Zammit 2012).

With given values of the fracture toughness and crack shape coefficient, a relationship between the critical stress and the flaw size is defined in eq. 3. However, an alternative expression is suggested in the following, with support of the experimental findings above.

Eq. (2) shows that the critical stress is inversely proportional to the square root of the mirror radius size. It may be used to express a relationship with the critical flaw size using eq. (1). As this relationship is similar to eq. (3) above, equating the stress terms in eq. (3) and in eq. (2), one obtains: 


$$
\sigma \sqrt{r_{M}}=\frac{K_{I C} \sqrt{10}}{Y \sqrt{\pi}}=A
$$

Thus, eq. 4 yields the ratio $K_{I C} / Y$ that is expressed in terms of the constant $A$. This new formulation that is introduced in the present version of the model may be advantageous as it bypasses the need to determine the parameter $Y$ of the flaw shape, and does include its exact effect through the constant:

$$
\frac{K_{I C}}{Y}=0.56 A
$$

This expression has another important advantage that is discussed in section 4.4.

\subsection{The distribution of the flaw sizes}

Many studies bypass the flaw distribution issue, and prefer to focus on the distribution of the measured ultimate capacity and fit a predefined known common statistical function to represent this distribution. The present model considers the flaws in glass as a fundamental property of the glass plate that governs its behaviour, and therefore a flaw distribution map is required. There is only limited information about the distribution of flaws in glass and this is insufficient to provide a general flaw map for implementation in the model. Therefore, a general flaw distribution model that is based on logical considerations has been hypothesized (Yankelevsky 2014); the flaw density and the size of the largest flaw are considered as the key characteristic parameters of the model. The model is used to distribute flaws in a basic glass plate with area $A_{0}$ that is produced and shipped, before being cut into the considered specimens (Yankelevsky 2014). Thus, cutting out specimens from the basic plate assigns a different flaw map to each specimen that is taken out of the global flaw map. For the present analysis of the behaviour of new glass, a flaw density of $1 \mathrm{flaw} / \mathrm{cm}^{2}$ is assumed. This complies 
with limited data from a recent preliminary study (Wereszczak 2014), where the measured density of flaws in soda lime glass varied between $1.18-2.60$ flaws $/ \mathrm{cm}^{2}$. The flaw distribution model is described in detail in (Yankelevsky 2014).

\subsection{Algorithm to identify the OF location}

The flaws are distributed over the surface of the basic glass plate according to the abovedescribed flaw distribution function, to produce the global flaw map of the basic plate. A random selection of the location of each specimen is assigned and the cut-out specimen dimensions and location in the basic plate are specified to determine the specific specimen flaw map that is generated from the global flaw map. For a given flaw map, of a given specimen, a corresponding map of the critical stresses may be produced using eq. 3 .

At this point the aspect of the crack orientation should be discussed. Clearly the orientation of a crack with respect to the global coordinate system that is aligned with the specimen length and width should be considered in addition to the flaw size and location. The critical stress that is perpendicular to the flaw axis is related to the critical bending stress in the specimen through stress transformation from the crack local coordinate system to the global coordinate system. Lack of probabilistic information on crack orientation requires assumptions of a statistical orientation function and its incorporation into the analysis (Beason 1980; Norhuda et al. 2010). This introduces an extra degree of freedom in the present analysis. To avoid this, eq. 4-a can be used instead of eq. 3. This equation incorporates the flaw shape and the critical stress intensity factor, as explained in section 4.2, but also correlates the flaw size with the bending stress that is the stress in the longitudinal specimen direction. That means that the parameter A (eq. 4) correlates the critical flaw size with the tensile bending stress in the specimen span direction, and therefore already includes 
the transformation effect of the critical stress in its direction perpendicular to the crack axis to the critical bending stress in the direction of the applied bending stresses. The obtained experimental value for $\mathrm{A}$, with its rather small standard deviation, thus promises a reliable representation of the glass behaviour.

Subcritical crack growth causes the flaw population size to be time-dependent as it is affected, among other factors, by loading rate and humidity. In the present study all tests have been conducted according to a certain standard and all test conditions strictly comply with the standard requirement including the specified loading rate and environmental conditions of temperature and humidity. The parameter $A$ is determined from this test series and the following model analysis refers to these same conditions, to simulate these test results in general terms to gain the required insight. Thus, the parameter $\mathrm{A}$ already includes the aspects of subcritical crack growth for this series of tests and there is no need to consider any further subcritical crack growth aspects in this study.

The present model compares the applied bending stresses that are calculated from the given specimen geometry, boundary conditions and loading, with the critical bending stresses at the flaw locations. This determines the OF location and the corresponding stress, from which the maximum bending stress, maximum bending moment and critical applied load are determined. This is a straight-forward and fast procedure that is demonstrated in the following section. A Monte Carlo simulation is carried out for a large sample size with many specimens and the accumulated results are gathered and analyzed. 


\section{Model Predictions}

This section simulates and analyzes the experimental series of tests with model predictions of the test data, in order to gain further insight into the examined specimens. The following analysis covers the major failure characteristics that are described above.

a. The Location of the Origin of Fracture - The measured longitudinal coordinate of the OF from the support (Figure 5) is compared with the model-predicted distribution of the OF locations (Figure 10). As expected, the model analysis for a sample of 2000 specimens yields an almost constant density along the specimen's central region between the loads, where a constant bending moment exists. However, failure also occurs in the shear span zone with a decreasing density towards the supports. This phenomenon is also observed in the experimental program where a smaller experimental sample was used.

The model sample size (2000 specimens) is rather large and is therefore expected to yield an almost converged prediction. Indeed, a repeated analysis with another randomized flaw sample of the same sample size yields similar results. This is not the case for a small sample of 56 specimens. Figure 11 shows some 14 repeated model analyses of simulated samples of 56 specimens each and their comparison with the test data.

Each model prediction of a 56-specimen sample looks quite different from another sample, with no clear governing rule behind the different distributions. The distributions are not symmetrical as well as having occurrences within the shear spans and their proximity to the supports. This indicates that a 56 -specimen size sample is too small to yield a clear unique distribution. All distributions, both model-predicted and experimental, are characterized by density fluctuations of higher and lower densities along the span. In general, the model predicts a range of OF locations that is similar to the experimental range. The envelopes of the predicted results are marked in Figure 11. It shows that the experimental cumulative 
distribution is bounded by the different computed cumulative distributions. This indicates that a simulation can yield a result close to the experimental data. All model predictions in Figure 11 indicate a rather linear trend of growth, of similar slope. This result could be expected for the model prediction of the large sample, showing a constant density along most of the span, but could not be predicted beforehand as a result of the fluctuating OF density variation that is observed in Figure 5. It turns out that the integrated result of the fluctuating OF density follows the expected trend, which finally converges to the large sample result.

It may be argued that experimental samples of 56 specimens are too small to provide a representative result for the examined glass panes, and there is no doubt that another experimental series of 56 specimens will yield different results. A considerably larger sample is required to yield converged experimental results that are significantly different than the above results, as can be visualized in comparison with the results of the large sample of 2000 specimens (Figure 10).

b. $\quad$ The Critical Tensile Bending Stress at Fracture - The distribution of the tensile bending stress at failure is shown in Figure 6. Figure 12 shows a comparison with the model prediction of the strength distribution of a sample of 5000 specimens. The model prediction has a clear bell-shaped distribution, as opposed to the apparently disordered distribution of the experimental sample of 56 specimens.

The mean tensile stress at failure for a sample of 5000 specimens is $82.91 \mathrm{MPa}$, which is slightly larger than the average experimental tensile stress (79.63 $\mathrm{MPa}$ ) of the 56 specimens. A rather wide range of strength is predicted, similar to the experimental observation.

The model was used once again to predict some 30 samples of 56 specimens each. Every prediction yielded another different disordered density distribution of the tensile bending stress at failure. Figure 13 shows a comparison of the cumulative probability of the 
experimental test data with and the cumulative probability curves of all predicted 56specimen-size samples.

Figure 13 shows the scatter of small size samples that form a band of predicted results. The experimental test data falls within this band and indicate that the model can predict a result that is similar to the test data. The mean stress of the experimental sample is located at about the mid-width of the prediction band.

c. Size of Critical Flaw - Fractographic measurements of the mirror radius, from which the size of the critical flaw was derived, have been described above. The model was also implemented to predict the critical flaw size distribution. Figure 14 shows the critical flaw size distribution that was predicted by the model for a sample of 5000 specimens and its comparison with the experimentally derived distribution.

The model predicts a similar range of critical flaw radii and, despite the experimental density fluctuations due to the small sample size, both distributions show a similar trend. It can be concluded that there is a good correspondence between the density distributions of the experimental data and model predictions, for critical flaw size.

\section{Discussion and Conclusions}

A series of strength tests on standard soda lime glass plate specimens has been carried out under controlled conditions. The specimens were subjected to a four-point loading system. Only specimens with face failure damage were considered, and edge failure cases were eliminated. Test data show that the location of the OF varies considerably, along most of the specimen span. There was no connection with the location of maximum bending moment. Also, the appearance of the cracking pattern and the fracture is highly variable too. The 
tensile stress at failure varied widely between $53 \mathrm{MPa}$ and $129 \mathrm{MPa}$ and the stress density distribution did not show a clear trend. Microscopic examination of the fractured region of each specimen provided a means to assess the critical flaw size. A wide range of flaw radii was found, varying between 23-178 $\mu \mathrm{m}$.

A recently developed theoretical stochastic model was implemented to predict the test series and further analyze the fracture behaviour. The model showed good predictions of fracture characteristics. It enables analysis of any sample size and provided a clear bellshaped stress distribution for a sufficiently large sample, and generated different predictions for limited size samples. Analysis of the experimental sample size of 56 specimens shows significant differences between different simulations of same-size samples. It is found that relatively small sample sizes that are commonly used in standard tests inherently yield different results with a pronounced variation. The standard's recommendation for a minimum sample size of 30 specimens is therefore too small to provide a significant and repeatable result.

The experimental study confirmed the empirical relationship between the mirror radius of the fractured glass and the tensile stress at failure. A constant value " $A$ " was determined having a small standard deviation. This parameter may successfully replace the fracture toughness and crack shape parameters in the critical flaw fracture expression.

The hypothesized flaw distribution model that has been used in the stochastic model is substantiated by the good correlation with the flaw size data. The seemingly random nature of fracture in annealed glass sample tests has been shown herein to be attributable to the distribution of flaws within the glass. By using parameters for typical flaw sizes and their scatter, in the suggested flaw distribution model, it is shown that the observed variation in 
glass fracture location can be very well estimated by the model. The experimental results obtained lie within the range of the numerically predicted results for a particular sample size. The proposed stochastic model yields good predictions of both the mean tensile stress at failure and its cumulative distribution.

\section{Acknowledgements}

The authors are grateful for the financial support of Explora Foundation, the Natural Sciences and Engineering Research Council of Canada (NSERC) and the Lyon Sachs Collaborative Research Fund.

\section{References}

ASTM C158-02. 2012. Standard test methods for strength of glass by flexure (Determination of modulus of rupture). American Society for Testing and Materials, West Conshohocken, PA.

Beason, W.A. 1980. Failure prediction model for window glass. PhD thesis, Texas Tech University, Lubbock, TX.

Beason, W.L., and Morgan, J.R. 1984. Glass failure prediction model. ASCE, Journal of Structural Engineering, 110(2): 197-212.

Brown, W.G. 1972. A load duration theory for glass design. National Research Council of Canada, Division of Building Research, Ottawa, ON.

Dalgliesh, W.A., and Taylor D.A. 1990. The strength and testing of window glass. Canadian Journal of Civil Engineering, 17(5): 752-762. 
EN 572-1. 2004. Glass in building - Basic soda lime silicate glass products - Part 1: Definitions and general physical and mechanical properties. European Committee for Standardization, Brussels, Belgium.

EN 1288-3. 2000. Glass in building - Determination of the bending strength of glass Part 3: Test with specimen supported at two points (four point bending). European Committee for Standardization, Brussels, Belgium.

Griffith, A. 1920. The phenomena of rupture and flow in solids. Royal Society of London, 221: 163-198.

Irwin, G. 1957. Analysis of stresses and strains near the end of a crack traversing a plate. Journal of Applied Mechanics 24: 361-364.

Irwin, G. 1962. Crack-extension force for a part-through crack in a plate. Journal of Applied Mechanics, 29: 651-654.

Lawn, B. 1993. Fracture of brittle solids. Second Edition, Cambridge University Press, Cambridge, UK.

Ledbetter,S.R., Walker, A.R., and Keiller,A.P. 2006. Structural use of glass. ASCE, Journal of Architectural Engineering, 12(3): 137-149.

Leon, J.A. 2001. New design model based on actual behavior of glass panels subjected to wind load. Glass Processing Days [online]. Available from http:// www.glassfiles.com

Marshall, D.B., Lawn, B.R., and Mecholsky, J.J. 1980. Effect of residual contact stresses on mirror/flaw-size relations. Journal of the American Ceramics Society, 63(5-6): 358360. 
Mechnolsky, J.J., Rice, R.W., and Freiman, S.W. 1974. Prediction of fracture energy and flaw size in glasses from measurements of mirror size. Journal of the American Ceramics Society, 57(10): 440-443.

Norville, H. S., Bove, P.M., Sheridan, D.L., and Lawrence, S.L. 1993. Strength of new heat treated window glass lites and laminated glass units. ASCE, Journal of Structural Engineering, 119(3): 891-901.

Nurhuda, I., Lam, N.T.K., Gad, E.F., and Calderone, I. 2010. Estimation of strengths in large annealed glass panels. International Journal of Solids and Structures, 47: 25912599.

Overend, M., and Zammit, K.A. 2012. Computer algorithm for determining the tensile strength of float glass. Engineering Structures, 45: 68-77.

Sedlacek, I.G., Blank, K., and Gusgen, J. 1995. Glass in structural engineering. The Structural Engineer, 17(2): 17-22.

Veer, F.A., and Rodichev, Y.M. 2011. The structural strength of glass: hidden damage. Strength of Materials, 43(3): 302-15.

Wereszczak, A.A., Ferber, M.K., and Musselwhite, W. 2014. Method for identifying and mapping flaw size distributions on glass surfaces for predicting mechanical response. International Journal of Applied Glass Science, 5(1): 16-21.

Yankelevsky D.Z. 2014. Strength prediction of annealed glass plates - A new model. Engineering Structures, 79: 244-255. 
Table 1: Test results for four-point bending specimens (Face Failure)

\begin{tabular}{|c|c|c|c|c|c|}
\hline $\begin{array}{c}\text { Test } \\
\text { Number }\end{array}$ & $\begin{array}{l}\text { Failure } \\
\text { Stress } \\
\text { (MPa) }\end{array}$ & $\begin{array}{l}\text { Fracture } \\
\text { Location } \\
\text { (mm from } \\
\text { end 1) }\end{array}$ & $\begin{array}{l}\text { Mirror } \\
\text { Radius, } r_{M} \\
(\mathrm{~mm})\end{array}$ & $\begin{array}{l}\text { Crack Half } \\
\text { Length, b } \\
(\mathrm{mm})\end{array}$ & $\begin{array}{c}\mathrm{A} \\
\left(\mathrm{MPa}^{1 / 2} \mathrm{~m}^{1 / 2}\right)\end{array}$ \\
\hline 1 & 2 & 3 & 4 & 5 & 6 \\
\hline 1 & 82.5 & 95 & 0.523 & 0.052 & 1.89 \\
\hline 2 & 98.9 & 143 & 0.425 & 0.043 & 2.04 \\
\hline 3 & 89.3 & 179 & 0.594 & 0.059 & 2.18 \\
\hline 4 & 71.4 & 147 & 0.655 & 0.066 & 1.83 \\
\hline 5 & 59.8 & 122 & 0.902 & 0.090 & 1.80 \\
\hline 6 & 57.0 & 137 & 1.122 & 0.112 & 1.91 \\
\hline 7 & 85.3 & 102 & 0.579 & 0.058 & 2.05 \\
\hline 8 & 78.1 & 94 & 0.637 & 0.064 & 1.97 \\
\hline 9 & 77.2 & 170 & 0.681 & 0.068 & 2.01 \\
\hline 10 & 88.6 & 125 & 0.551 & 0.055 & 2.08 \\
\hline 11 & 95.9 & 168 & 0.449 & 0.045 & 2.03 \\
\hline 12 & 94.3 & 141 & 0.474 & 0.047 & 2.05 \\
\hline 13 & 81.4 & 94 & 0.738 & 0.074 & 2.21 \\
\hline 14 & 67.2 & 141 & 0.990 & 0.099 & 2.12 \\
\hline 15 & 92.6 & 113 & 0.425 & 0.043 & 1.91 \\
\hline 16 & 69.5 & 160 & 0.390 & 0.039 & 1.78 \\
\hline 17 & 64.5 & 89 & 1.299 & 0.130 & 2.33 \\
\hline 18 & 59.4 & 88 & 1.294 & 0.129 & 2.14 \\
\hline 19 & 69.3 & 117 & 0.894 & 0.089 & 2.07 \\
\hline 20 & 79.9 & 103 & 0.720 & 0.072 & 2.14 \\
\hline 21 & 87.9 & 128 & 0.607 & 0.061 & 2.16 \\
\hline 22 & 102.6 & 135 & 0.390 & 0.039 & 2.03 \\
\hline 23 & 78.8 & 114 & 0.639 & 0.064 & 1.99 \\
\hline 24 & 74.4 & 113 & 0.772 & 0.077 & 2.07 \\
\hline 25 & 78.2 & 93 & 0.709 & 0.071 & 2.08 \\
\hline 26 & 76.3 & 117 & 0.796 & 0.080 & 2.15 \\
\hline 27 & 55.3 & 148 & 0.733 & 0.073 & 1.50 \\
\hline 28 & 70.3 & 140 & 0.872 & 0.087 & 2.08 \\
\hline 29 & 68.9 & 89 & 0.820 & 0.082 & 1.97 \\
\hline 30 & 63.9 & 102 & 1.130 & 0.113 & 2.15 \\
\hline 31 & 56.5 & 80 & 1.451 & 0.145 & 2.15 \\
\hline 32 & 86.8 & 87 & 0.590 & 0.059 & 2.11 \\
\hline 33 & 85.6 & 133 & 0.856 & 0.086 & 2.50 \\
\hline 34 & 68.6 & 130 & 1.007 & 0.101 & 2.18 \\
\hline 35 & 82.0 & 122 & 0.605 & 0.060 & 2.02 \\
\hline 36 & 74.9 & 121 & 0.787 & 0.079 & 2.10 \\
\hline 37 & 129.2 & 122 & 0.232 & 0.023 & 1.97 \\
\hline 38 & 109.7 & 168 & 0.388 & 0.039 & 2.16 \\
\hline 39 & 99.4 & 180 & 0.407 & 0.041 & 2.01 \\
\hline
\end{tabular}


Table 1-continued: Test results for four-point bending specimens (Face Failure)

\begin{tabular}{|c|c|c|c|c|c|}
\hline $\begin{array}{c}\text { Test } \\
\text { Number }\end{array}$ & $\begin{array}{c}\text { Failure } \\
\text { Stress } \\
(\mathrm{MPa})\end{array}$ & $\begin{array}{c}\text { Fracture } \\
\text { Location } \\
(\mathrm{mm} \text { from } \\
\text { end 1) }\end{array}$ & $\begin{array}{c}\text { Mirror } \\
\text { Radius, } \mathrm{r}_{\mathrm{M}} \\
(\mathrm{mm})\end{array}$ & $\begin{array}{c}\text { Crack Half } \\
\text { Length, b } \\
(\mathrm{mm})\end{array}$ & $\begin{array}{c}\mathrm{A} \\
\left(\mathrm{MPa}-\mathrm{m}^{1 / 2}\right)\end{array}$ \\
\hline 1 & 2 & 3 & 4 & 5 & 6 \\
\hline 40 & 95.2 & 110 & 0.443 & 0.044 & 2.00 \\
41 & 67.7 & 174 & 1.048 & 0.105 & 2.19 \\
42 & 72.4 & 90 & 0.711 & 0.071 & 1.93 \\
43 & 57.1 & 121 & 1.414 & 0.141 & 2.15 \\
44 & 70.7 & 104 & 0.760 & 0.076 & 1.95 \\
45 & 77.4 & 74 & 0.686 & 0.069 & 2.03 \\
46 & 112.2 & 157 & 0.351 & 0.035 & 2.10 \\
47 & 109.9 & 93 & 0.316 & 0.032 & 1.95 \\
48 & 73.7 & 140 & 0.881 & 0.088 & 2.19 \\
49 & 83.8 & 173 & 0.572 & 0.057 & 2.01 \\
50 & 53.5 & 168 & 1.516 & 0.152 & 2.08 \\
51 & 85.9 & 73 & 0.596 & 0.060 & 2.10 \\
52 & 82.5 & 123 & 0.617 & 0.062 & 2.05 \\
53 & 85.9 & 90 & 0.520 & 0.052 & 1.96 \\
54 & 87.9 & 76 & 0.534 & 0.053 & 2.03 \\
55 & 79.3 & 131 & 0.630 & 0.063 & 1.99 \\
56 & 53.0 & 77 & 1.784 & 0.178 & 2.24 \\
\hline
\end{tabular}




\section{List of Symbols}
A constant
a shear span
b half length of the critical crack
$K_{\text {I }}$ stress intensity factor
$\mathrm{K}_{\mathrm{Ic}}$ critical stress intensity factor
I span
P load
$r_{F}$ critical flaw radius
$r_{M}$ mirror radius
Y geometrical constant
$\sigma$ stress 


\section{Figure Captions}

Figure 1: Mirror region and other zones in a typical glass fracture surface

Figure 2: Test setup - four-point bending (dimensions in $\mathrm{mm}$ )

Figure 3: Load frame and safety box

Figure 4-a: Cross-section of a specimen with a typical face failure

Figure 4-b: Cross-section of a specimen with a typical edge failure

Figure 5: Location of fracture origins in four-point bending tests (supports at coordinates 0 and $20 \mathrm{~cm}$; loads at coordinates 5 and $15 \mathrm{~cm}$ )

Figure 6: Distribution of tensile strength at fracture in four-point bending tests

Figure 7: Typical views of crack patterns

Figure 8: Close-up at the fracture origin region in a test specimen

Figure 9: Distribution of critical flaw radii as derived from test series

Figure 10: Location of fracture origins in four-point bending - comparison of model prediction with test data

Figure 11: Model predictions of OF locations (56 specimens)

Figure 12: Cumulative probabilities of test and predicted OF location - 56 specimen samples

Figure 13: Comparison of model prediction with experimental tensile strength

Figure 14: Model vs. test tensile bending stress at failure (sample size=56) 


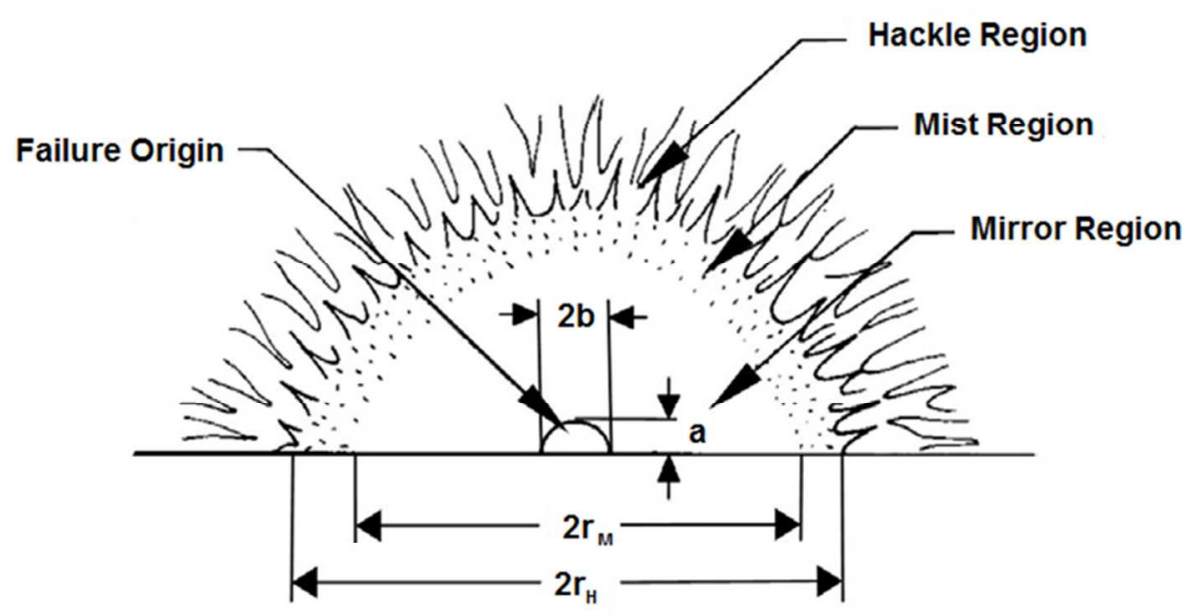

$254 \times 190 \mathrm{~mm}(96 \times 96$ DPI) 


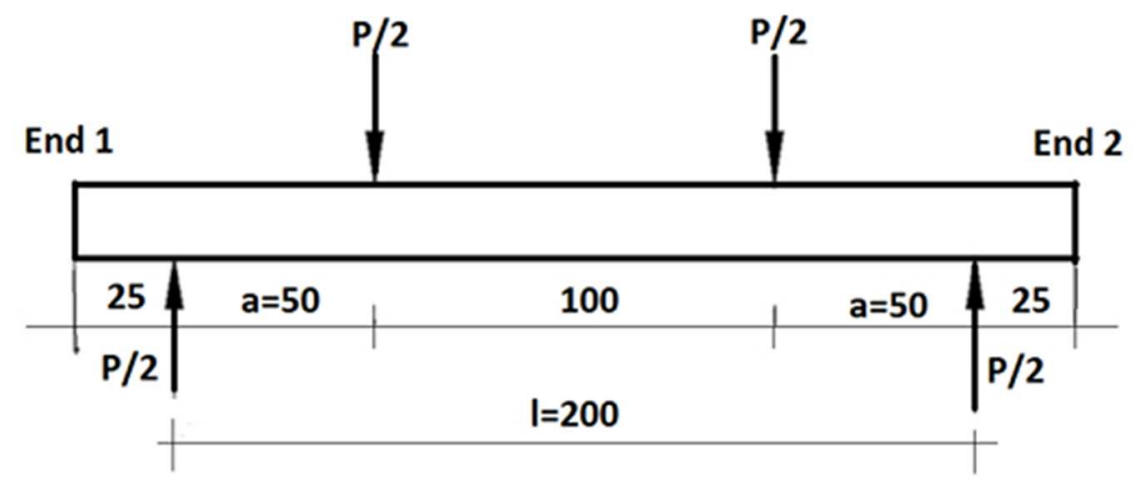




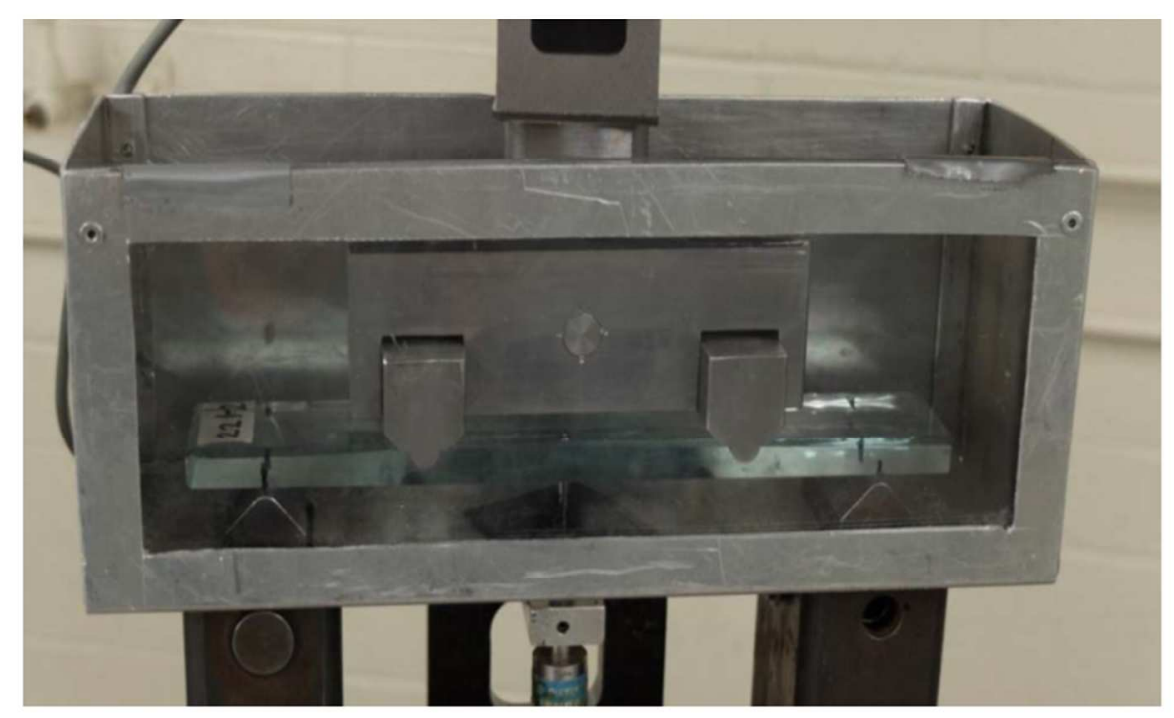

$254 \times 190 \mathrm{~mm}(96 \times 96$ DPI $)$ 


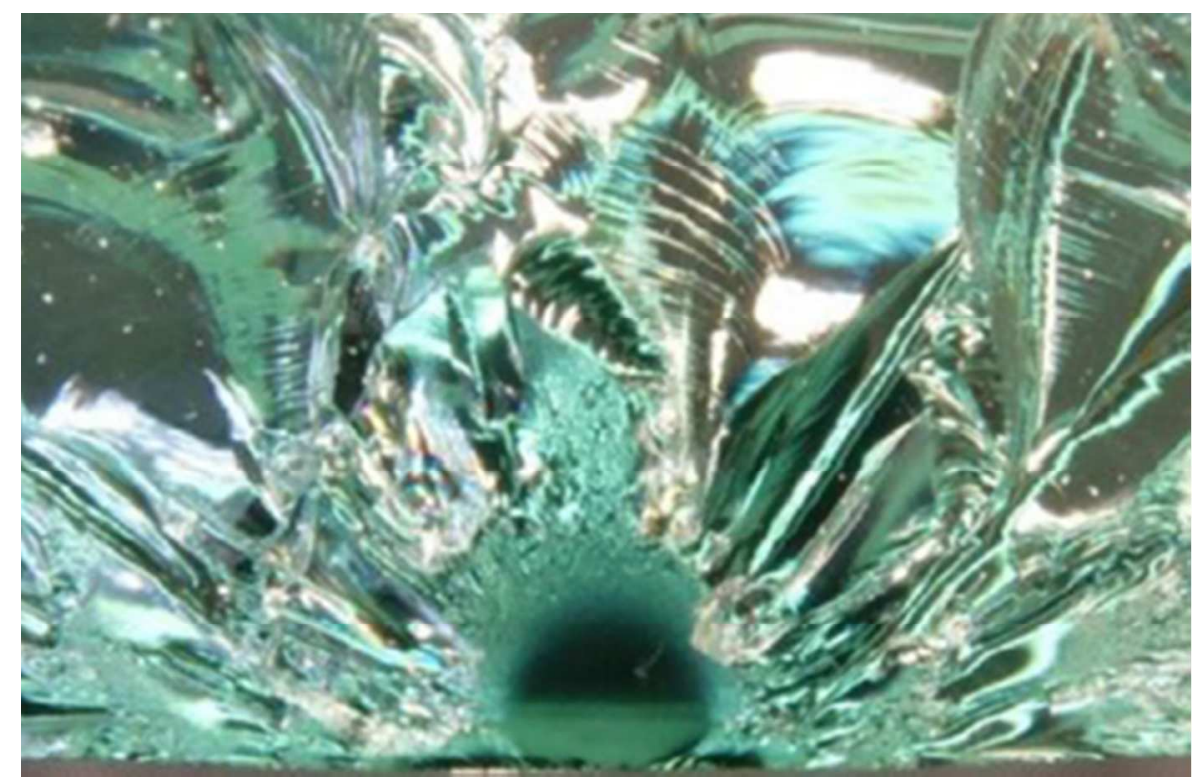




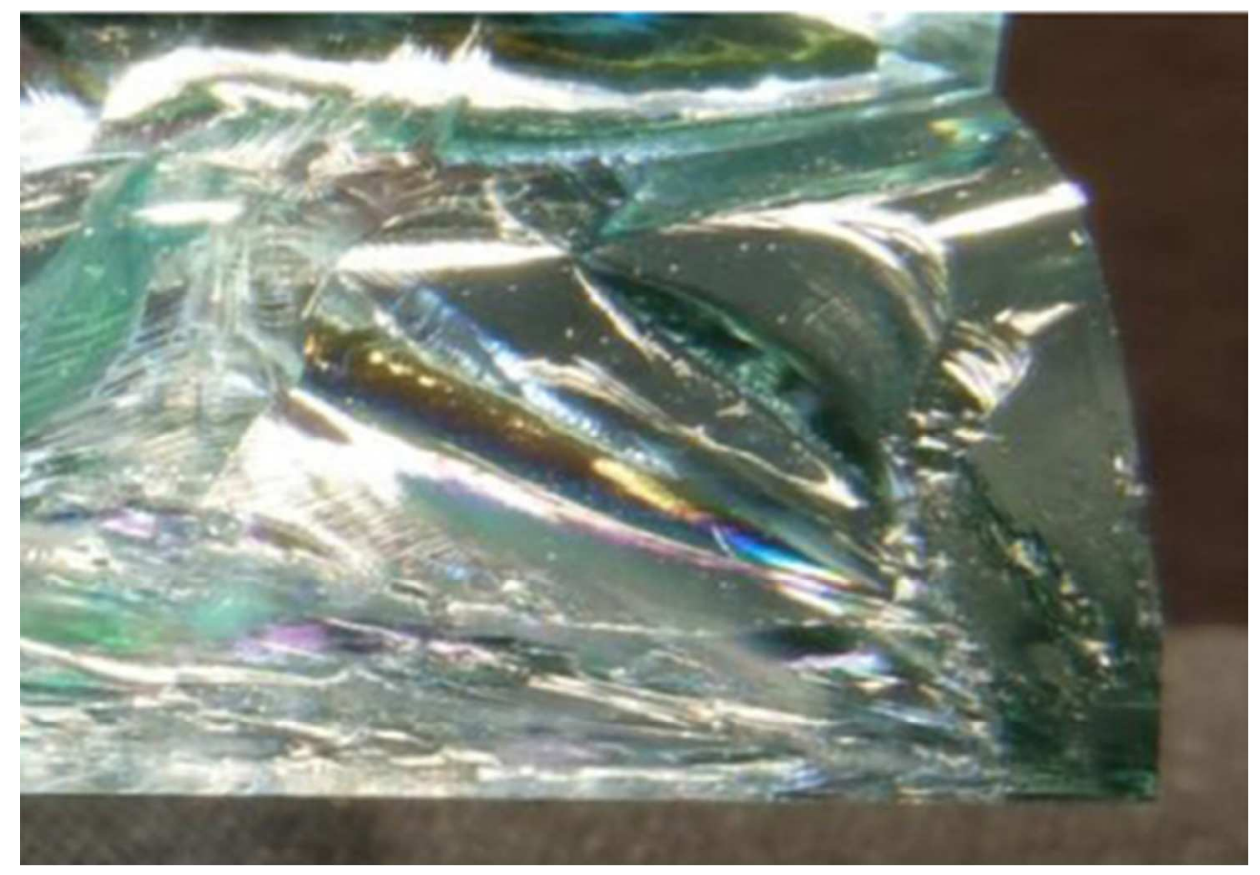




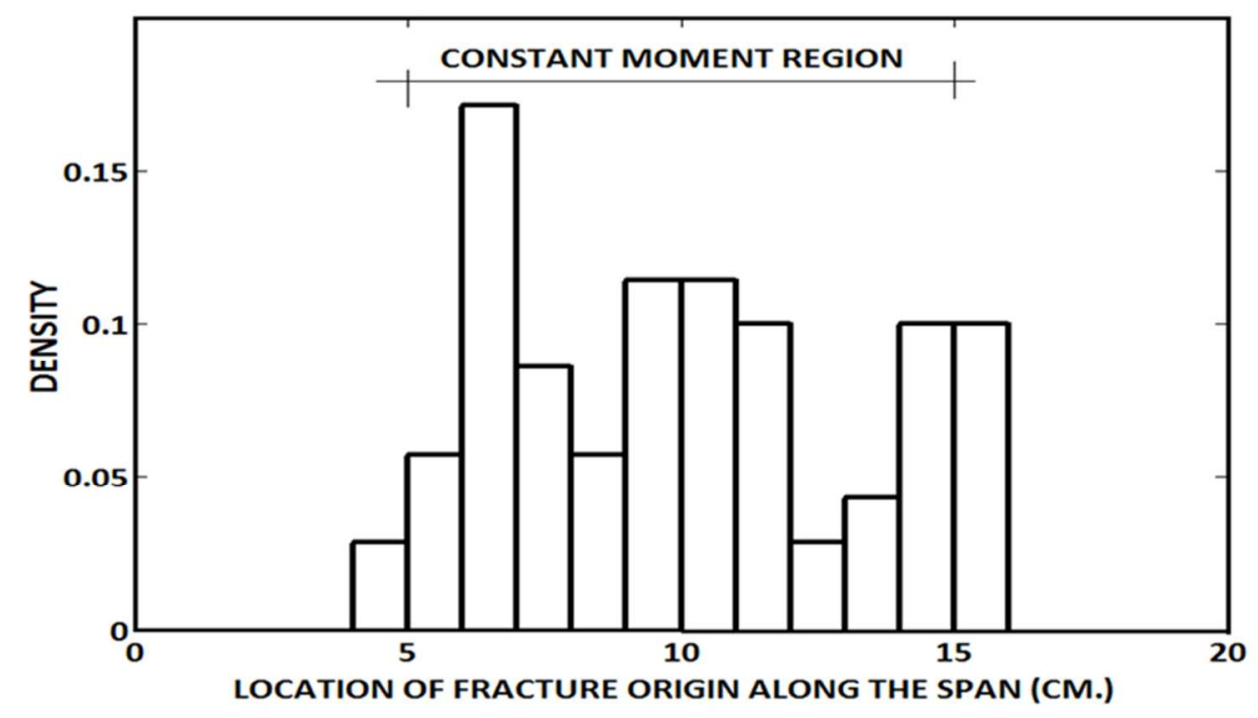

https://mc06.manuscriptcentral.com/cjce-pubs 


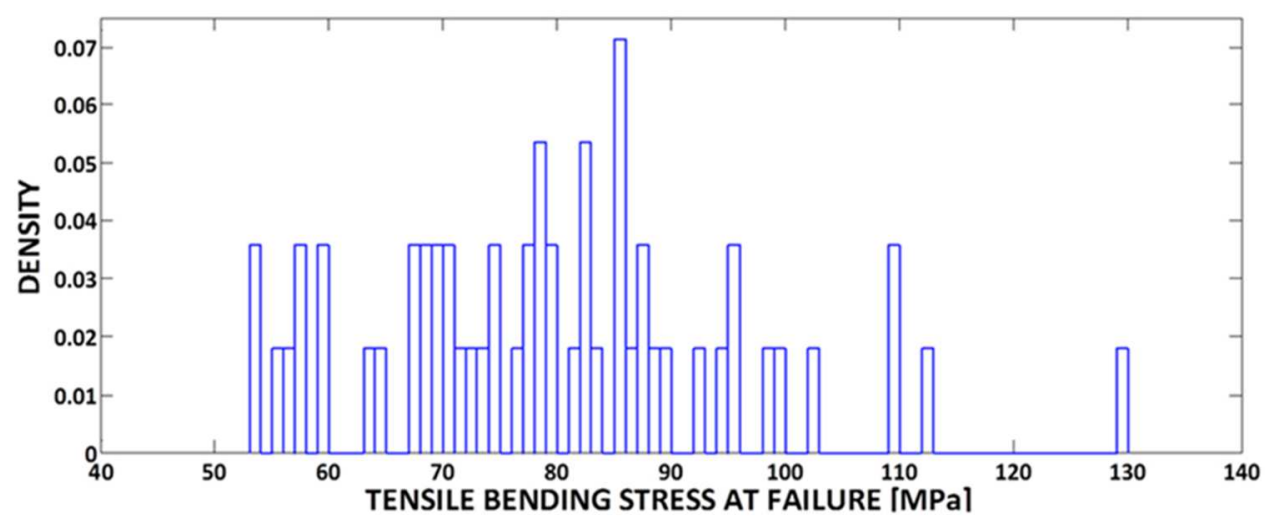

https://mc06.manuscriptcentral.com/cjce-pubs 


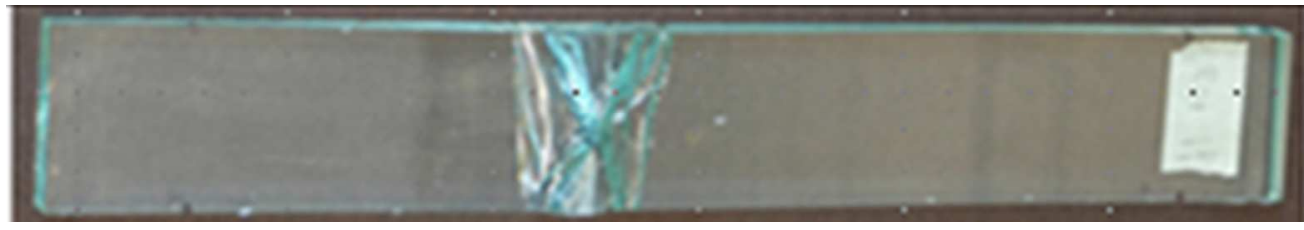

https://mc06.manuscriptcentral.com/cjce-pubs 


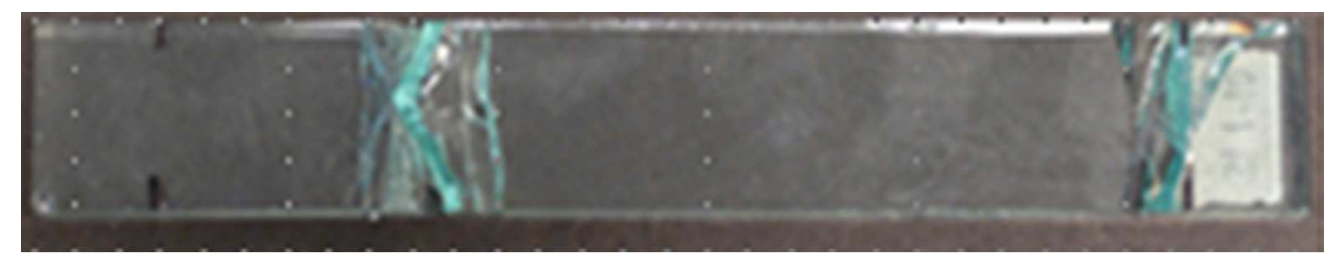

https://mc06.manuscriptcentral.com/cjce-pubs 


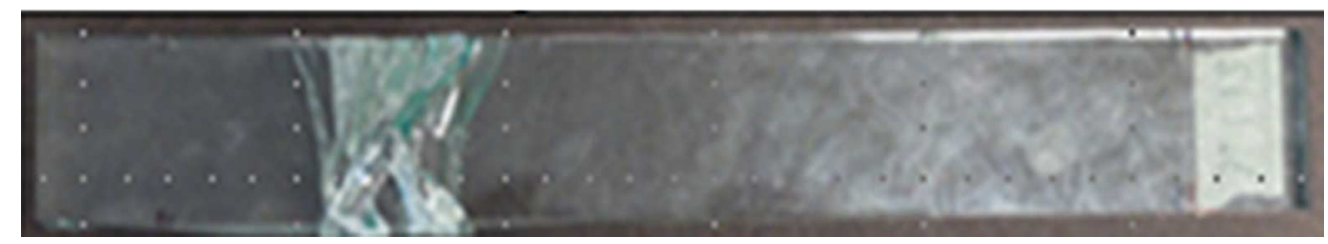

https://mc06.manuscriptcentral.com/cjce-pubs 


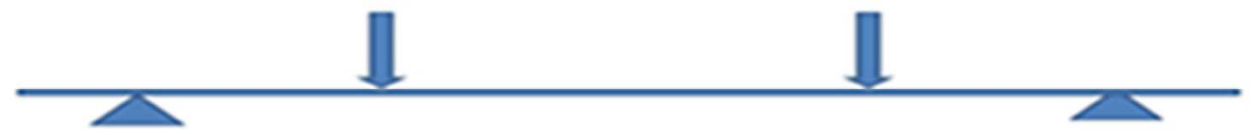




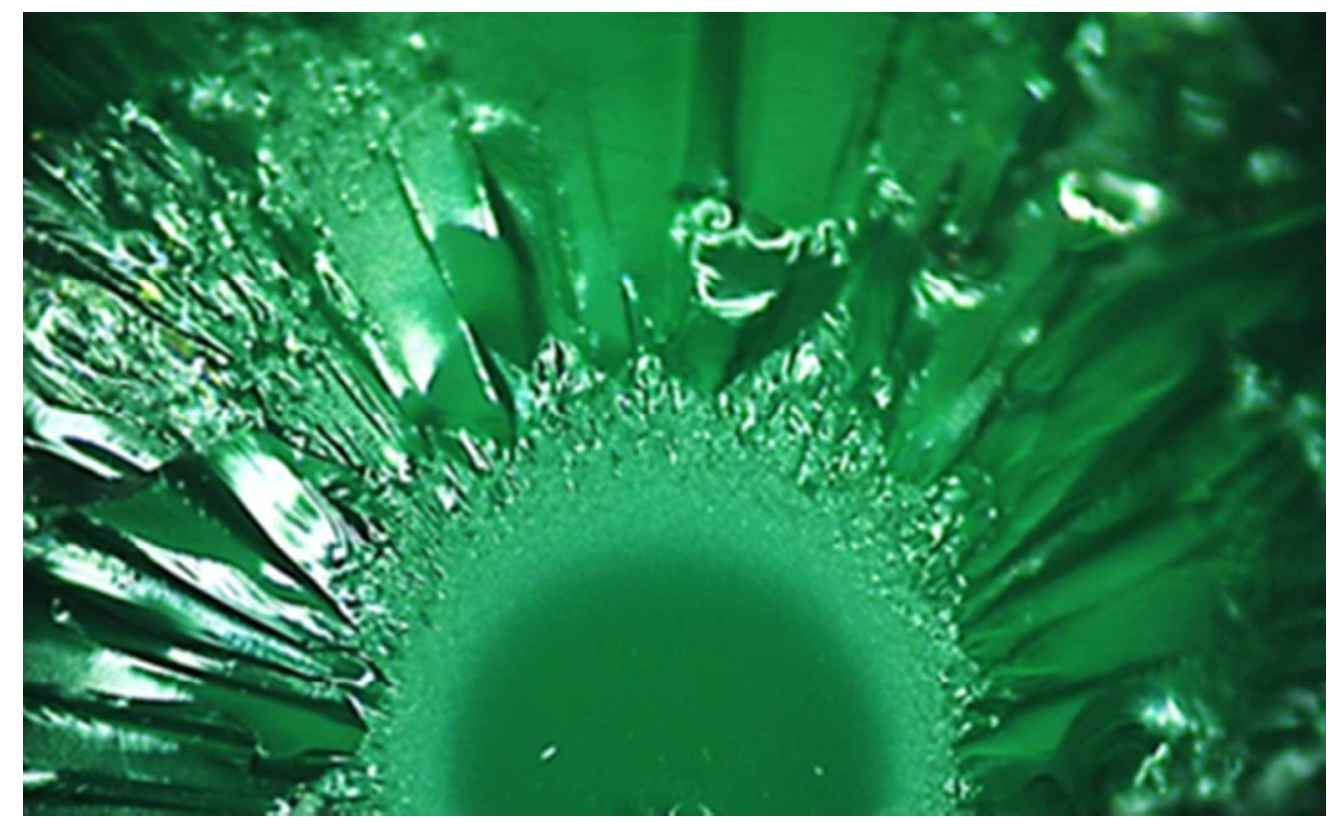




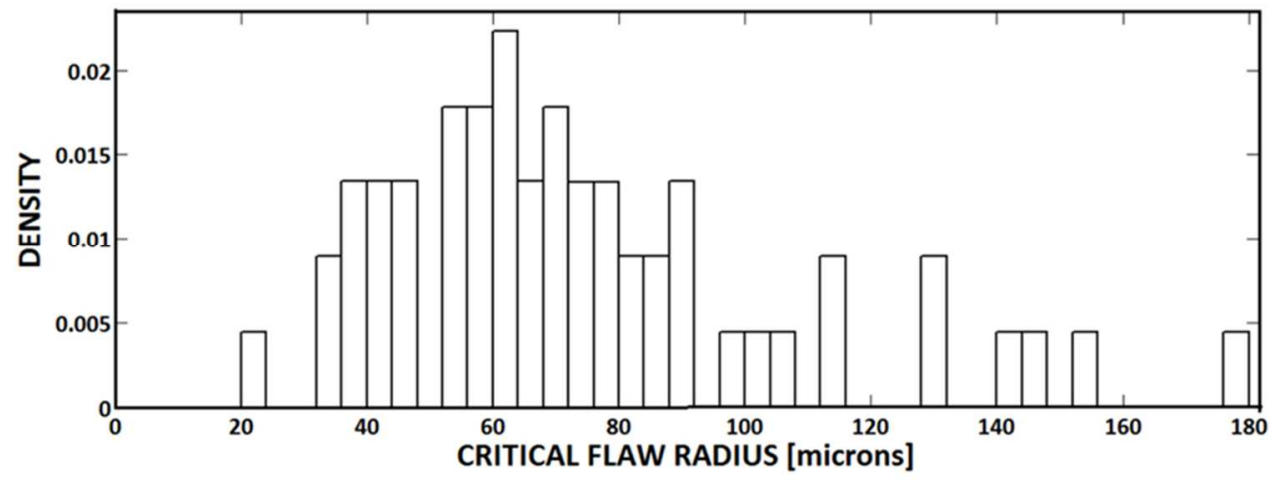

https://mc06.manuscriptcentral.com/cjce-pubs 


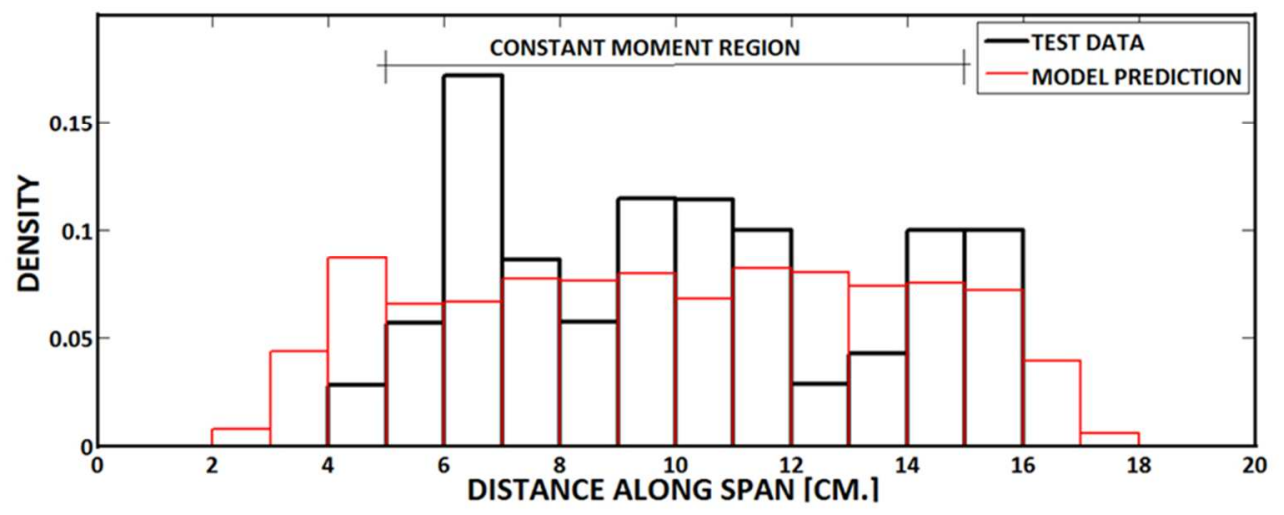




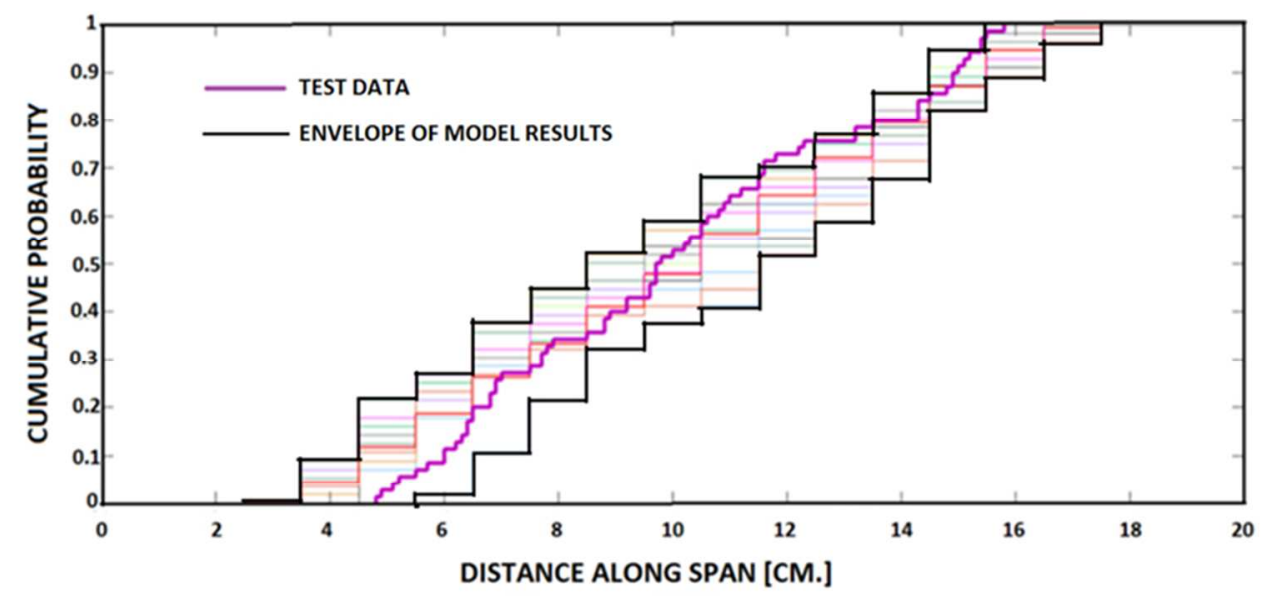

https://mc06.manuscriptcentral.com/cjce-pubs 


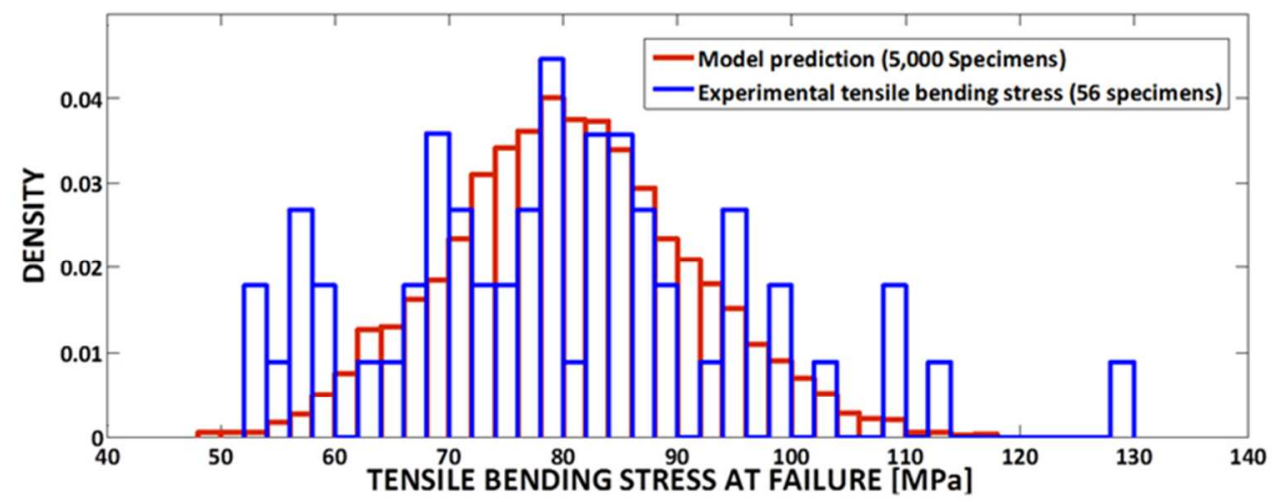

https://mc06.manuscriptcentral.com/cjce-pubs 


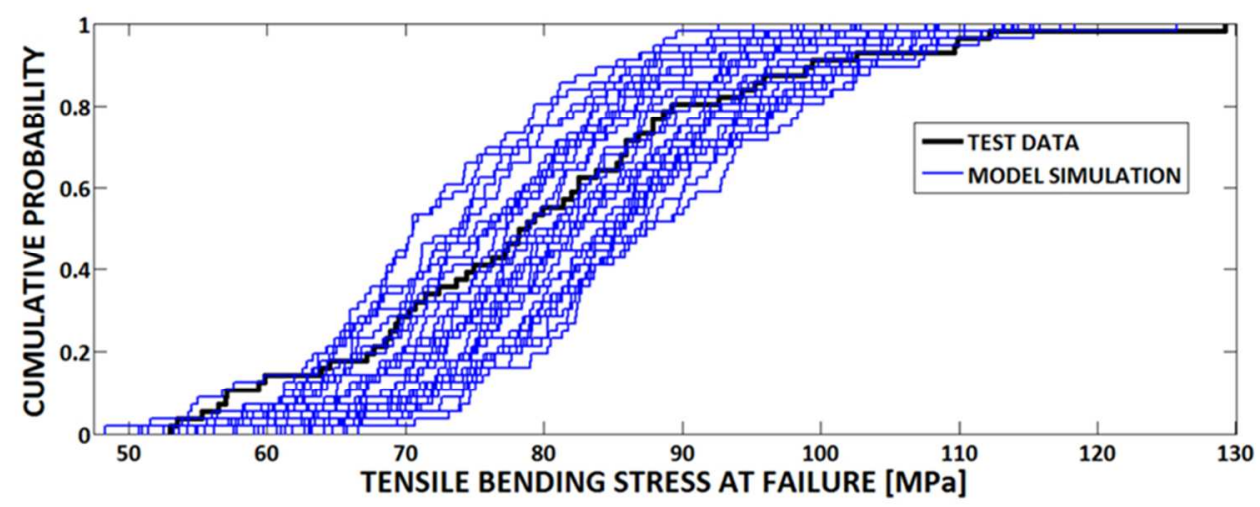

https://mc06.manuscriptcentral.com/cjce-pubs 


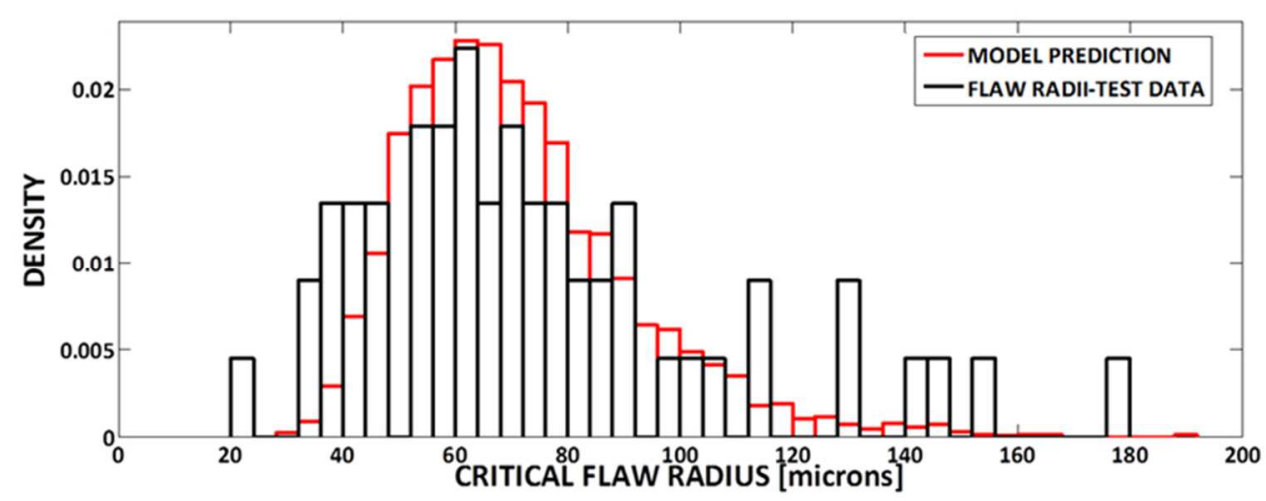

https://mc06.manuscriptcentral.com/cjce-pubs 